for patients. There is an education need across the region to raise awareness and confidence of digital legacy, which the undertaking authors will be addressing.

\section{IS SIMULATION AN EFFECTIVE WAY TO TEACH PALLIATIVE MEDICAL EMERGENCIES TO SPECIALIST TRAINEES?}

Samuel Krauze, Fiona Wiseman. Northampton General Hospital NHS Trust, Northamptonshire Healthcare NHS Foundation Trust

\subsection{6/bmjspcare-2018-ASPabstracts. 12}

Background Simulation is increasingly being used to train those in 'acute' medical specialties, offering the chance to practice in a safe environment, without compromising patient safety. It lends itself particularly to infrequently occurring situations which are potentially life-threatening. Its use in palliative medicine is mainly limited to advanced communication skills - there is little published work regarding its use for acute clinical skills.

Methods A simulation day was arranged for eleven palliative medicine specialist trainees in the East Midlands. This covered five scenarios (hypoglycaemia, opioid toxicity, acute left ventricular failure, massive haemorrhage and anaphylaxis) from the specialty training curriculum for palliative medicine. In each scenario trainees took part in pairs, the 'patient' being an actor, or the 'SimMan' manikin. There was a nurse and HCA present in each, and an actor playing a relative was present in three of the scenarios. Each was observed by the consultant present. The trainees not taking part watched events unfold via video-link. Feedback was led by the consultant present, with input from an acute medic. There was group discussion with all trainees present. The consultant completed 'mini-CEXs' for each trainee in the scenario.

Results Pre and post-simulation day feedback was collected via a $1-5$ scale ( $1=$ strongly disagree, $5=$ strongly agree), and showed:

They felt their clinical knowledge increased (3.5 vs 4.0)

They felt their confidence increased (3.6 vs 4.1 )

They felt they would learn/had learnt something new from the day (4.3 vs 4.7$)$

Free text feedback explained that trainees felt it was a fun and effective way to learn, and that it was especially beneficial to have other members of the multidisciplinary team present, and to receive feedback from acute medics.

Conclusion Simulation seems to be an acceptable, enjoyable, and effective way to teach palliative medical emergencies. Trainees are keen for simulation to be incorporated into their training.

\section{Free papers 13-15 | New models of care}

\begin{tabular}{l|l}
\hline 13 & OPTIMISING THE MANAGEMENT OF PATIENTS WITH \\
CANCER PAIN: DEVELOPMENT AND EVALUATION OF \\
THE RAPID ACCESS MULTIDISCIPLINARY PALLIATIVE \\
ASSESSMENT AND RADIOTHERAPY (RAMPART) CLINIC
\end{tabular}

Andrew Jenks, Paul Fenton, Carol Davis, Charlotte Brooks, Lynsey Clode, Inga Driver, Mark Cawley. University Hospital Southampton NHS Foundation Trust
Background Patients with cancer-induced bone pain often wait weeks to receive palliative radiotherapy treatment and to be assessed by specialist palliative care and allied health services. While waiting, they experience debilitating physical problems and psychological distress. This paper reports on the development and evaluation of RAMPART, a 'one-stop' multidisciplinary clinic at University Hospital Southampton. This innovation has not previously been reported in the UK.

Methods The clinic model involves a single visit and combines assessment by palliative medicine, clinical oncology and allied health professionals, with the planning and delivery of palliative radiotherapy. The intervention also involves signposting, onward referrals and supported self-management of physical, psychological and social concerns. A patient satisfaction questionnaire and Macmillan's Holistic Needs Assessment are performed on the clinic day and repeated one month later. Open response questions are asked on the day and at 1 month.

Results Overall, $87 \%$ of patients were very satisfied and 13\% were satisfied. Patients' global concern score decreased by 1.9 points, mean score 7.1 (range 4-10) on clinic day to 5.2 (range 2-8) at 1 month. There was a reduction in pain score by 2 points, mean score 6.8 (range $3-10$ ) on clinic day to 4.8 (range $0-8$ ) at 1 month. The RAMPART clinic model successfully reduced the median time from referral to radiotherapy from 22 days in the comparator cohort to 8 days in the RAMPART cohort. Qualitative data findings are that patients felt supported, enlightened, informed and valued by the comprehensive nature of the assessment. Patients felt their symptoms and quality of life had improved.

Conclusions Implementing a multidisciplinary palliative radiotherapy clinic is feasible, valued by patients and effective in reducing pain, other patient concerns and time from referral to treatment. This model helps to bridge the gap between hospital and community services and may be transferrable to other areas.

\section{ENHANCED SUPPORTIVE CARE - JOINT WORKING BETWEEN SUPPORTIVE CARE AND ACUTE ONCOLOGY TO DELIVER RAPID ACCESS TO EXPERTISE}

Lisa La Mola, Geraldine Campbell, Richard Berman, Tim Cooksley, Tamar Al-Sayad, Hannah Clare. The Christie NHS Foundation Trust

\subsection{6/bmjspcare-2018-ASPabstracts. 14}

The Enhanced Supportive Care (ESC) clinic provides patients with better access to supportive care services. It allows any Christie patient with problems relating to their cancer, or cancer treatment, to be seen without delay. This helps to reduce the escalation of symptoms and medical problems that could potentially lead to hospital admission. ESC clinic is a new joint service provided by Supportive Care and Acute Oncology teams.

A six month pilot has demonstrated a reduction in emergency admissions to the Christie, a reduction in patients sent elsewhere within Greater Manchester (GM), a reduction in the need for GP follow up appointments, reduced length of stay and has facilitated early discharges from the Oncology Assessment Unit (OAU), which improves patient flow.

Referrals into the ESC clinic are received from multiple areas, namely:

- Acute Oncology Management Service/Hotline 
- Oncology patients from Outpatient areas

- Radiotherapy treatment areas

- Oak Road Treatment Centre.

- Community Macmillan direct referrals

A bespoke proforma was completed after each consultation by the treating clinician. 413 ambulatory patient reviews took place over a 6 month period. An in depth analysis has been conducted of 236 new patient referrals between January and June 2017. Overall results show a significant impact on patient outcomes, with $21 \%$ avoided admissions, 25\% facilitated early discharge and $43 \%$ avoided escalation of symptoms/GP review.

Data analysis remains on going to assess readmission statistics; in particular an analysis of early OAU discharges, facilitated by the ESC clinic review of low risk neutropenic patients.

The service currently operates two clinics a week on Monday and Thursday afternoons. A business case has been submitted proposing service expansion to operate daily clinics.

As the first cancer centre to implement this service, we have welcomed oncology teams from other centres across the UK, enhancing practice elsewhere.

\section{AN EVALUATION OF PALLIATIVE CARE NURSE PRESCRIBING: A MIXED METHODS STUDY IN UGANDA}

J Downing, G Kivumbi, E Nabirye, A Ojera, R Namwanga, R Katusabe, M Dusabimana, K Kalema, B Yayeri, A Apollo, M Batuli, C Komunda, R Nabukalu, J Mwesige, M Sekyondwa, M Kasirye, JO Amoris, E Nandutu, W Acuda, D Adong, E Luyirika, E Namisango, F Kiyange, R Kiwanuka, J Amandua, J Logan, E Haraldsdottir, B Moback, C Lesley, L Grant, M Leng. Makerere and Mulago Palliative Care Unit Makerere University Kampala, Global Health Academy University of Edinburgh, Dr Ambrosoli Memorial Hospital Agago, Hospice Africa Uganda Kampala, Kabale Hospital Kabale, Bwera Hospital Kasese, CARO Kasese, MJAP Bwizibwera Health Centre Mbarara, Palliative Care Association of Uganda, Kampala, Kibuli Hospital Kampala, Kagadi Hospital Kibale, Mildmay Uganda, Management Sciences for Health Busia, Tororo Hospital, Uganda People's Defence Force (UPDF) Bombo, African Palliative Care Association Kampala, Ministry of Health Kampala, St Columba's Hospice Edinburgh, Cairdeas International Palliative Care Trust UK

\subsection{6/bmjspcare-2018-ASPabstracts. 15}

Background Access to palliative care (PC) in Uganda has been hampered by the lack of prescribers. Thus PC care trained nurses have been able to prescribe since 2004, however, no full scale evaluation had been undertaken, despite the fact that PC stakeholders are advocating for nurse prescribing in other countries. Thus an evaluation was undertaken to determine the effectiveness of PC nurse prescribing in Uganda.

Methodology The mixed methods evaluation was undertaken in three parts:

- Preparation of the nurses for the role;

- Assessing and managing pain \& symptoms;

- An appraisal of the system in which the nrses are working.

Ethical approval was gained prior to the evaluation commencing.

Results

- Training: the curriculum prepares the nurses well, however, a few adaptions are recommended to strengthen it further.

Themes identified from 18 interviews included: the training itself, supervision and mentorship, compentency, and beliefs.

- Assessing and managing pain: 22 nurses were recruited to care for 20 PC patients each, visiting them on at least 2 occassions, assessing and managing their pain and other symptoms, and utilising the APCA POS. Analysis demonstrates nurses are able to assess and manage pain, giving appropriate mediciations and reassessing.

- The appraisal in 10 districts, showed nurses are working in a system which can impact negatively on care provision e.g. stck-outs of analgesics, limited understanding of PC generally: and many myths exist re the use of morphine and provision of PC.

Trained palliative care nurses can assess and manage patients pain, prescribing morphine and associated medications appropriately. Whilst challenges exist, recommendations are made to improve the system, this evaluation has clearly shown the benefit and safety of nurse prescribing for PC in Uganda. This study has important implications for the global debate on issues of access to oral morphine and task shifting.Conclusion

\section{Free papers 16-18 | Communication}

\section{WHAT VALUE AND CONTRIBUTION CAN ARCHAEOLOGY GIVE TO END-OF-LIFE PRACTITIONERS?}

Karina Croucher, Christina Faull, Laura Green, Lindsey Büster, Jennifer Dayes. University of Bradford, LOROS Hospice

\subsection{6/bmjspcare-2018-ASPabstracts. 16}

Background What happens when archaeology meets health and social care? The Continuing Bonds Study, a pilot project lead by Dr Karina Croucher, Professor Christina Faull and Laura Green, uses case studies of the dead from the recent and distant past to spark discussions about death and dying. Via a series of workshops, the study investigates what value and consequence such materials and discussions have for health and social care students and practitioners.

Methods In this qualitative, exploratory study, 92 participants attended 21 workshops delivered in Bradford and Leicester. Themed case studies presented materials in picture, video and object formats accompanied by explanatory text. Responses to pre, post and follow-up (1-3 months) questionnaires have been analysed both descriptively and thematically. Recordings of the discussions generated in the workshops were also analysed thematically.

Results Our results confirm the project hypothesis, with 93\% of survey respondents believing that archaeological materials can be used to facilitate discussions/training about death, dying, bereavement and loss. For some individuals, the followup questionnaire identified considerable personal and professional impacts of the workshop. $81 \%$ of the respondents remembered at least one archaeological example, effected by how interesting and emotive they perceived the material. The project had a further result, with $72 \%$ saying the workshops helped to reflect on differences in cultural attitudes to death and dying. Some individuals feel more confident talking about bereavement post-workshop, and now give further consideration to patients' choices in the context of dying.

Conclusions Archaeological materials facilitate discussions around death, dying and bereavement that are relevant to health and social care workers, personally and professionally. Participants welcomed the opportunity to discuss and explore the myriad aspects of death from different times and places. 